INRA Prod. Anim., 2011, 24 (4), 369-376

\title{
Perspectives d'application de la sélection génomique dans les schémas d'amélioration génétique porcins
}

T. TRIBOUT

INRA, UMR1313 Génétique Animale et Biologie Intégrative, F-78352 Jouy-en-Josas, France AgroParisTech, Génétique Animale et Biologie Intégrative, 16 rue Claude Bernard, F-75231 Paris, France

Courriel : Thierry.Tribout@jouy.inra.fr

L'évaluation génomique est une nouvelle méthode d'estimation de la valeur génétique des animaux d'élevage, et son implémentation dans les schémas de sélection bovins laitiers a permis d'améliorer fortement leur efficacité. La récente disponibilité d'une puce porcine de 60000 marqueurs SNP permet aujourd'hui d'envisager une sélection génomique chez le porc. Cet article analyse l'organisation des schémas de sélection porcins actuels, et indique les voies possibles d'amélioration grâce à cette nouvelle méthodologie.

Au cours des 20 dernières années, de nombreux QTL (Quantitative Trait Loci, ou locus à effet quantitatif) ont été mis en évidence dans l'ensemble des espèces animales de rente. Des programmes de Sélection Assistée par Marqueurs (SAM) ont été mis en place avec succès dans plusieurs espèces, en France par exemple chez les bovins laitiers (Fritz et al 2003) ou encore chez les ovins pour la résistance à la tremblante (Sidani et al 2010). Dans l'espèce porcine en revanche, force est de constater que jusqu'à ce jour les applications de SAM ont été très peu nombreuses (Van der Steen et al 2005, Luther et al 2009, Schwob et al 2009), en dépit des centaines de QTL localisés (Hu et Reecy 2007). Cette situation s'explique principalement par le fait que les marqueurs microsatellites (seuls marqueurs disponibles jusqu'à récemment) sont généralement en équilibre de liaison avec les QTL au niveau d'une population (obligeant à raisonner intra-famille ce qui complique les programmes de SAM), et éloignés des mutations causales (d'où une diminution rapide de l'efficacité de la sélection en raison des recombinaisons entre marqueurs et QTL).

$\mathrm{Au}$ cours des dernières années, un nouveau type de marqueurs - les marqueurs SNP («Single Nucleotide Polymorphism») - répartis sur 1'ensemble du génome et en nombre extrêmement élevé (plusieurs centaines de milliers à plusieurs millions de mar- queurs sur l'ensemble d'un génome) a été mis en évidence. Grâce au développement de puces à SNP, on peut aujourd'hui génotyper pour un faible coût (moins de $200 €$ ) un individu pour plusieurs dizaines (voire centaines) de milliers de ces marqueurs. Parallèlement à ces avancées, des travaux ont montré qu'il était possible, en subdivisant le génome en un très grand nombre de segments définis par un ou plusieurs marqueurs SNP, d'estimer précisément la valeur génétique d'un individu comme la somme des effets de ces segments, l'effet de chaque segment étant au préalable estimé sur un groupe d'individus pour lesquels on dispose à la fois du phénotype et du génotype aux marqueurs.

Cette nouvelle méthodologie d'évaluation, appelée «sélection génomique», est en train de révolutionner les schémas de sélection bovins laitiers depuis quelques années. Elle permet en effet de sélectionner avec une grande précision les jeunes mâles dès leur naissance sans avoir besoin de les soumettre à un testage sur descendance long et coûteux, et d'augmenter les intensités de sélection sur les voies mâles et femelles (Guillaume et al 2011).

Chez le porc, la disponibilité depuis 2009 d'une puce porcine de 60000 SNP ouvre de nouvelles perspectives d'utilisation d'information des marqueurs dans les dispositifs d'améliora- tion génétique. La présente communication a pour objet d'évaluer l'intérêt de la sélection génomique pour l'espèce porcine et d'aborder les difficultés liées à une éventuelle mise en œuvre de cette méthodologie.

\section{1 / Organisation actuelle des schémas d'amélioration génétique porcins ; forces et faiblesses}

Chez le porc, les dispositifs d'amélioration génétique ont une structure pyramidale, en général à 3 étages (figure 1). $\mathrm{Au}$ sommet de cette pyramide se trouvent les élevages de sélection, dans lesquels on trouve les populations de race pure et les lignées synthétiques. Ces élevages de sélection approvisionnent les élevages des autres niveaux de la pyramide et les centres d'insémination artificielle en reproducteurs de race pure ou de lignées synthétiques. Au niveau intermédiaire se trouvent les élevages de multiplication dans lesquels on produit des cochettes et des verrats croisés à partir d'animaux nés à l'étage de sélection. Ces animaux croisés sont vendus aux élevages de production, qui constituent le dernier niveau de la pyramide, afin de produire les porcs charcutiers (résultats du croisement entre une femelle croisée (la «truie parentale») et un verrat parental croisé ou de race pure). 
Figure 1. Organisation générale des dispositifs d'amélioration génétique porcins.

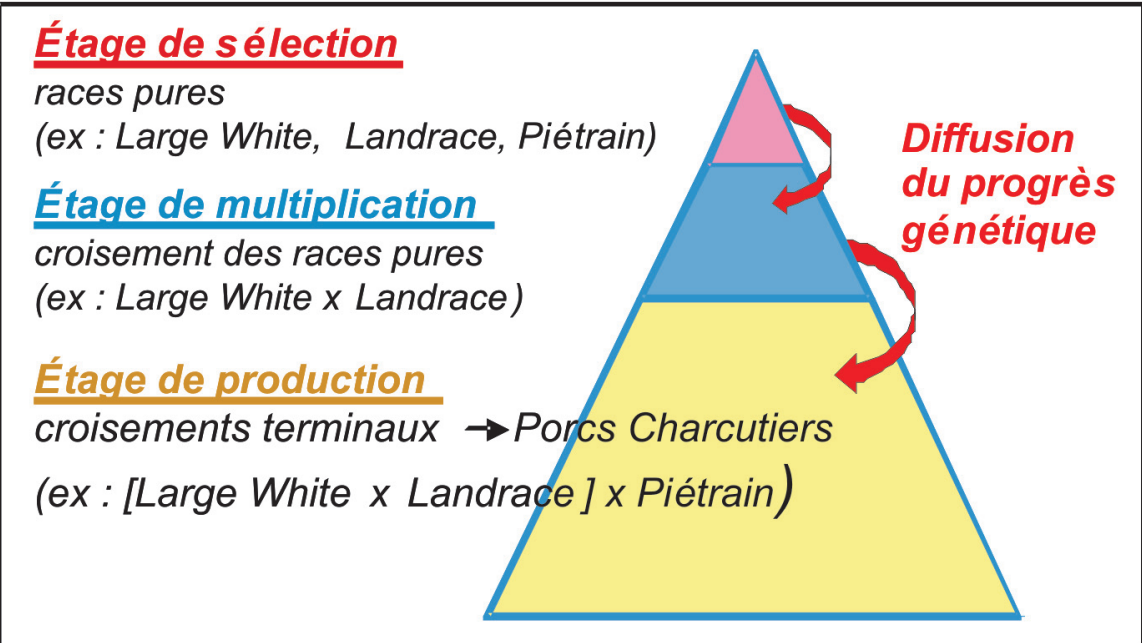

Cette organisation pyramidale s'accompagne d'une spécialisation des populations de l'étage de sélection. Schématiquement, on y trouve des populations maternelles sélectionnées principalement pour améliorer les caractères de prolificité et d'aptitudes maternelles (qui sont combinées en multiplication pour produire des truies parentales croisées), et des populations paternelles sélectionnées pour améliorer les caractères d'engraissement et la valeur de la carcasse (utilisées comme verrat terminal en race pure ou après croisement en multiplication).

L'ensemble du travail d'amélioration génétique est réalisé à l'étage de sélection sur les populations de race pure et les lignées synthétiques ; l'objectif final est cependant d'améliorer les performances de reproduction et d'aptitudes maternelles des truies parentales croisées et les performances d'engraissement et de qualité de la carcasse des porcs charcutiers terminaux des élevages de production. Cette concentration de l'effort de sélection au sommet de la pyramide est fondée sur le principe que le progrès génétique qui y est réalisé se transmet jusqu'à l'étage de production.

A l'exception du schéma bavarois s'appuyant en partie sur un testage sur descendance (Habier et al 2007), les dispositifs d'évaluation génétique porcins actuels reposent sur un contrôle en élevage des performances des jeunes candidats mâles et femelles pour quelques caractères d'engraissement et de composition de la carcasse; ces mesures sont parfois complétées par le contrôle en station puis l'abattage d'un nombre limité de collatéraux des candidats, permettant de disposer de performances pour des caractères trop complexes ou trop coûteux à mesurer en élevage (efficacité alimentaire) ou enre- gistrés post mortem (caractères de qualité de la viande, composition de la carcasse...). Dans les populations maternelles, les performances de reproduction des truies (prolificité, aptitudes maternelles) sont également considérées. Les valeurs génétiques sont estimées grâce à la méthodologie du BLUP (Best Linear Unbiased Predictor, ou meilleur prédicteur linéaire non biaisé) appliquée à un modèle animal. Chaque individu (jeune candidat mâle ou femelle, reproducteur) dispose ainsi d'une valeur génétique estimée pour tous les caractères de l'objectif de sélection de tères pour lesquels il ne dispose pas de phénotype propre. En général, seules les performances des individus de la population sélectionnée sont considérées dans l'évaluation génétique, mais certains schémas prennent également en compte des performances d'apparentés croisés.

Les décisions de sélection ou de réforme des candidats mâles et femelles sont prises dès la fin de leur contrôle de performances en ferme (un peu avant 6 mois d'âge), et les intensités de sélection collective Large White type femelle française par exemple, 1 candidate sur 11 est conservée pour la reproduction en sélection et 1 candidat sur 65 entre en Centre d'Insémination Artificielle (CIA) et sera utilisé en sélection; dans la population collective Piétrain française, ces taux sont de 1/16 et $1 / 50$, respectivement. Les individus retenus sont mis à la reproduction dès 8 mois d'âge, et leur carrière de reproducteur en sélection est courte, environ 1 an pour les mâles et 2 ans pour les femelles. Ce turnover rapide s'explique d'une part par le progrès génétique réalisé dans les populations, qui fait que la supériorité génétique des reproducteurs sa population, y compris pour les caraction sont fortes. Ainsi, dans la popula- en activité par rapport au niveau des meilleurs candidats s'amenuise rapidement, ce qui impose leur réforme et leur remplacement, et d'autre part par la volonté de limiter la descendance des verrats dans les élevages de sélection afin de préserver la variabilité génétique dans la population. En conséquence directe de ces pratiques, les intervalles de génération sont très faibles, tant sur la voie mâle que sur la voie femelle, et seules des carrières encore plus courtes pourraient les réduire davantage.

\section{Faiblesses des dispositifs actuels :}

Cette organisation des schémas d'amélioration porcins, bien que résultant en des intervalles de génération faibles et en des intensités de sélection fortes tous deux propices à un progrès génétique important, présente cependant plusieurs faiblesses.

Tout d'abord, la faible durée de carrière des reproducteurs limite le nombre de leurs produits phénotypés, et la précision d'estimation de leurs valeurs génétiques reste donc globalement faible. Ainsi, le Coefficient de Détermination (CD) moyen des verrats de CIA actifs dans les populations collectives françaises pour les caractères mesurés sur les individus contrôlés en station varie entre 0,18 pour le rendement technologique estimé (caractère le moins héritable, $\mathrm{h}^{2} \sim 0,28$ ) et 0,50 pour le taux de muscle des pièces (caractère le plus héritable, $\mathrm{h}^{2} \sim 0,75$ ), et se limite à 0,30 pour le nombre de porcelets nés vivants par portée $\left(\mathrm{h}^{2} \sim 0,10\right)$ dans les populations maternelles. Il en va de même pour le CD des valeurs génétiques des jeunes candidats en fin de contrôle de performances, en moyenne très modeste pour ces mêmes caractères $(0,10,0,40$ et 0,16 , respectivement). Cette précision limitée des valeurs génétiques estimées au moment de la sélection des reproducteurs de renouvellement pénalise le progrès génétique, les risques de sélectionner un individu qui se révèlera finalement mauvais étant non négligeables. Des schémas fondés sur un testage sur descendance des verrats (comme le schéma bavarois) permettraient certes de disposer de valeurs génétiques estimées plus précises et donc de diminuer les risques lors de la sélection des candidats, mais au prix cependant d'un allongement de l'intervalle entre générations et d'une réduction de l'intensité de sélection venant réduire le progrès génétique réalisé.

Comme évoqué précédemment, l'ensemble du travail d'amélioration génétique est réalisé à l'étage de sélection sur les populations de race pure et les lignées synthétiques, mais l'objectif réel est d'améliorer les performances des truies parentales croisées et des porcs 
charcutiers terminaux des élevages de production (qui représentent $95 \%$ de la population porcine globale). Le principe sous-jacent à cette organisation est que le progrès génétique réalisé à l'étage de sélection dans les populations de race pure et les lignées se transmet (avec un retard d'une ou deux générations) aux individus croisés de l'étage de production. Généralement, les valeurs génétiques des candidats et des reproducteurs des élevages de sélection sont estimées uniquement à partir des performances d'individus des races pures (ou des lignées) enregistrées en élevage ou dans des stations de contrôle. Cependant, ces performances peuvent parfois se révéler être des prédicteurs imparfaits de celles des descendants croisés élevés dans des conditions de production, pour deux raisons principalement. Tout d'abord, les individus de l'étage de sélection sont élevés dans des environnements extrêmement maîtrisés et d'excellent statut sanitaire, et ces conditions d'élevage favorables peuvent différer sensiblement des conditions de production. Or, les animaux les plus performants dans un milieu peuvent se révéler moyens voire médiocres dans un environnement différent. De telles interactions génotype $\mathrm{x}$ milieu ont par exemple été mises en évidence chez le porc par Merks (1989). Par ailleurs, des effets de dominance et d'épistasie inexistants dans deux races pures peuvent exister chez les individus issus de leur croisement. Ainsi, plusieurs auteurs rapportent des corrélations entre les performances d'individus de race pure d'élevages de sélection et celles de leurs descendants croisés comprises entre 0,3 et 1 selon les caractères et les populations considérées (Merks 1989, Lutaaya et al 2001, Habier et al 2007). En conséquence, le progrès génétique réalisé peut être sensiblement plus faible à la base de la pyramide qu'à son sommet.

Il est possible d'augmenter le progrès génétique réalisé en élevage de production en combinant les performances des individus de race pure à celles d'apparentés croisés lors de l'évaluation génétique des populations en sélection (Wei et Van der Steen 1991), et plusieurs schémas de sélection porcins se sont engagés dans cette voie. Cependant, la remontée d'informations en provenance des élevages de production s'avère en général difficile faute de généalogies de qualité suffisante et en l'absence de contrôle de performances. De plus, $1 \mathrm{ou}$ 2 générations (parfois plus dans le cas de croisements complexes) séparent les populations sélectionnées des porcs charcutiers, et sauf à vouloir organiser un testage sur descendance (qui augmenterait considérablement l'intervalle entre générations et pénaliserait le progrès génétique réalisé annuellement), le gain de précision obtenu pour les jeunes candidats grâce à leurs apparentés croisés serait minime.

\section{2 / Opportunités offertes par la sélection génomique dans l'espèce porcine}

Une puce pangénomique de 60000 marqueurs SNP est disponible chez le porc depuis 2009. Les résultats de Du et al (2007), de Uimari et Tapio (2011), ainsi que de récents sondages dans les principales populations porcines françaises en sélection (programme de recherche DELISUS ; Boitard communication personnelle) indiquent qu'avec une telle densité de marqueurs, le déséquilibre de liaison moyen $\left(\mathrm{r}^{2}\right)$ entre SNP adjacents serait supérieur à 0,20 (valeur de DL en-deçà de laquelle l'évaluation génomique ne semble pas efficace selon le retour d'expérience des schémas bovins laitiers français et étrangers); la mise en place d'une sélection génomique dans l'espèce porcine est donc envisageable. De plus, il est probable que des puces beaucoup plus denses seront disponibles dans les prochaines années, qui permettront de tirer parti d'associations encore plus fortes entre marqueurs et QTL. Cependant, avant de se lancer dans une telle évolution des schémas d'amélioration génétique porcins, il est impératif de se demander en quoi la sélection génomique pourrait améliorer leur efficacité.

\section{1 / Intervalle entre générations}

L'intervalle entre générations dans les dispositifs porcins actuels est très faible, de l'ordre de 2 ans dans les populations collectives françaises (Maignel et al 1998). Un changement des modalités d'évaluation génétique n'aurait qu'un impact très limité sur ce paramètre.

\section{2 / Intensités de sélection}

Dans un dispositif fondé sur l'évaluation génomique, chaque individu génotypé peut se voir calculer une valeur génomique, même s'il n'a pas de performance propre et ne dispose d'aucun apparenté phénotypé. Le choix des reproducteurs de renouvellement peut donc se faire en théorie parmi l'ensemble des porcelets nés, permettant de maximiser l'intensité de sélection et donc d'augmenter le progrès génétique réalisé. En pratique, le nombre de candidats dans un tel dispositif, et par conséquent l'intensité de sélection effective, seraient fonction des éventuels surcoûts liés au génotypage que les sélectionneurs seraient prêts à supporter compte tenu des gains génétiques supplémentaires attendus. Les intensités de sélection actuellement réalisées dans les schémas porcins sont cependant déjà élevées (le coût des mesures réalisées en ferme sur les candidats est faible, permettant de contrôler une forte proportion des porcelets nés), et le gain attendu en jouant sur ce paramètre pourrait donc ne pas être majeur.

\section{3 / Précision des valeurs géné- tiques estimées}

L'évaluation génomique peut en revanche avoir un impact beaucoup plus important sur l'efficacité des schémas par le biais d'une augmentation de la précision des valeurs génétiques estimées. Comme indiqué précédemment, les valeurs génétiques des candidats pour les caractères de reproduction (dans les lignées femelles) et pour les caractères mesurés sur collatéraux (caractères de qualité, d'efficacité alimentaire) sont estimées avec des précisions souvent modestes (CD moyens compris entre 0,10 et 0,40 selon le caractère). Le retour d'expérience des schémas de sélection génomique bovins laitiers et d'études réalisées par simulations (figure 2, établie d'après Meuwissen 2009) montrent qu'il serait possible d'estimer pour de jeunes candidats des valeurs génomiques plus précises que leurs valeurs génétiques actuelles, à la condition toutefois de disposer d'une population de référence de taille suffisante et de marqueurs couvrant le génome de manière suffisamment dense.

On constate cependant sur la figure 2 que l'obtention de $\mathrm{CD}$ supérieurs à 0,3 pour des caractères faiblement héritables (ce qui est le cas de la qualité de la viande, de la prolificité et des aptitudes maternelles par exemple) requerrait une population de référence d'environ 3000 à 5000 individus.

\section{4 / Sélection sur des caractères non mesurables en routine}

L'évaluation génomique pourrait également permettre de sélectionner les populations pour des caractères nouveaux, trop complexes ou trop coûteux à mesurer en routine (y compris dans le cadre d'un contrôle sur collatéraux) ou nécessitant la mise en place de protocoles expérimentaux spécifiques : mesures comportementales, résistance à des maladies, composition des rejets, maturité des porcelets à la naissance, homogénéité des porcelets intra-portée, odeurs sexuelles, qualité des aplombs, etc. On pourrait par exemple imaginer d'enregistrer dans quelques élevages équipés d'automates de pesée les poids des porcelets à la naissance et au sevrage pour un échantillon de truies génotypées; ces truies constitueraient une population de 
Figure 2. Précision théorique $(C D)$ des valeurs génomiques estimées en fonction de la taille de la population de référence et de l'héritabilité $\left(h^{2}\right)$ du caractère considéré, pour une population de taille efficace $(\mathrm{Ne})=100$ (d'après Meuwissen 2009).

On fait l'hypothèse que chaque QTL est en déséquilibre de liaison complet avec un marqueur SNP.

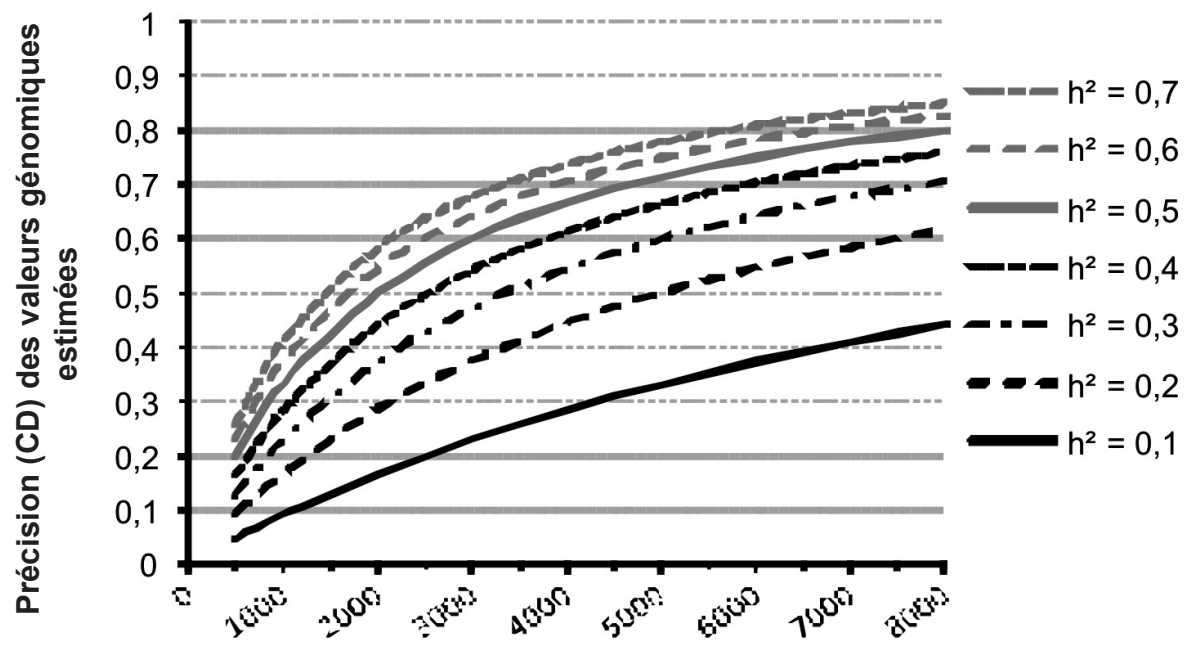

Nombre de phénotypes dans la population de référence

référence permettant d'estimer les valeurs génomiques des candidats à la sélection pour l'homogénéité du poids des porcelets intra-portée, la croissance des porcelets en période d'allaitement et la production laitière des truies, etc. Les coûts de génotypage et de phénotypage liés à la constitution initiale et au renouvellement périodique de telles populations de référence seraient cependant élevés, et ce type d'approche se limiterait vraisemblablement à quelques caractères parmi les plus importants pour la filière.

\section{5 / Augmentation du progrès génétique réalisé en production}

Comme évoqué précédemment, le fait de sélectionner des individus de race pure élevés dans un milieu très contrôlé dans le but d'améliorer les performances de leurs descendants croisés en conditions de production constitue l'une des principales faiblesses des dispositifs d'amélioration génétique porcins, et c'est probablement sur ce point que la sélection génomique pourrait présenter son plus grand intérêt chez le porc. Il serait en effet possible, en s'appuyant sur une population de référence constituée d'animaux croisés, idéalement phénotypés dans des conditions de milieu semblables à celles des élevages de production, d'estimer la valeur génomique de candidats de race pure pour des caractères exprimés par des animaux commerciaux (truies parentales ou porcs charcutiers). Dekkers (2007) et Kinghorn et al (2010) ont montré par simulation qu'une telle approche permettrait un progrès génétique plus important dans la population croisée qu'une sélection génomique s'appuyant sur une population de référence consti- tuée d'animaux de race pure. Elle serait également plus efficace qu'une sélection classique (BLUP-modèle animal) combinant les informations des individus de race pure et de leurs apparentés croisés (Dekkers 2007) sans nécessiter l'organisation d'un enregistrement en routine des généalogies et performances des descendants de production et sans augmenter l'intervalle entre générations.

Deux modèles ont été proposés pour estimer les effets des segments chromosomiques à partir d'une population de référence constituée d'animaux croisés (Ibanz-Escriche et al 2009, Kinghorn et al 2010). Pour décrire ces modèles, considérons par exemple que les individus de la population de référence sont issus du croisement entre une truie parentale croisée ayant des parents des races pures A et $B$ et un verrat terminal de race pure $C$, et que chaque segment chromosomique est défini par un seul marqueur biallélique.

Le premier modèle, le plus simple, fait l'hypothèse que l'effet du génotype à un marqueur sur la performance d'un animal croisé est identique quelles que soient les populations d'origine (A, B ou C) des allèles reçus par l'animal pour ce marqueur :

$$
y_{i}=\mu+\sum_{j} X_{i j} \beta_{j}+e_{i},
$$

où $y_{i}$ est la performance de l'individu croisé $i, \mu$ est la performance moyenne, $X_{i j}$ est le nombre d'allèles $1(0,1$ ou 2$)$ au locus du jème marqueur de l'individu $i, \beta_{j}$ est l'effet de substitution de l'allèle 1 au locus du jème marqueur dans la population de référence, et $e_{i}$ est le résidu pour l'individu $i$.

La valeur génomique estimée VGE d'un candidat q d'une quelconque des
3 races pures $\mathrm{A}, \mathrm{B}$ ou $\mathrm{C}$ se calcule alors simplement comme la somme sur l'ensemble des marqueurs considérés du produit du nombre d'allèles 1 portés par l'individu q au locus $\mathrm{j}\left(\mathrm{X}_{\mathrm{qj}}\right)$ par l'effet de substitution estimé pour cet allèle :

$$
V G E_{q}=\Sigma_{j} X_{q j} \beta_{j}
$$

Le second modèle considère au contraire que l'effet du génotype à un marqueur peut être différent en fonction de la population d'origine des allèles au marqueur :

$y_{i}=\mu+\Sigma_{j}\left(A_{i j k}^{P} \beta_{j k}^{P}+A_{i j l}^{M} \beta_{j l}^{M}\right)+e_{i}$

où $y_{i}$ est la performance de l'individu croisé i, $\mu$ est la performance moyenne, $A^{P}{ }_{i j k}$ est l'allèle porté par l'individu au jème SNP provenant de la race $k$ transmis par le père de l'individu (ici, l'allèle provient forcément de la race $\mathrm{C}$ puisque le père est de race pure), et $\beta^{P}{ }_{j k}$ est l'effet de substitution de l'allèle $A^{P}{ }_{i j k}$. De même, $A^{M}{ }_{i j l}$ est l'allèle porté par l'individu au jème SNP provenant de la race 1 transmis par la mère de l'individu (ici, l'allèle provient de la race A ou de la race $B$ puisque la mère de l'individu est croisée), et $\beta^{M}{ }_{j l}$ est l'effet de substitution de l'allèle $A^{M}{ }_{i j l}^{j l}$. Dans le cas d'une population croisée constituée d'individus $(\mathrm{A} \times \mathrm{B}) \times \mathrm{C}$, on estimera donc pour chaque SNP 3 effets : un effet $\beta^{P}{ }_{j C}$ pour l'allèle transmis par les pères, et deux effets $\beta^{M_{j A}}$ et $\beta^{M_{j E}}$ pour l'allèle transmis par les mères selon qu'il provient à l'origine de la population $\mathrm{A}$ ou $\mathrm{B}$, respectivement.

La valeur génomique des candidats de chacune des 3 races pures constituant le croisement est ensuite calculée à partir des effets des marqueurs estimés pour la race correspondante ; par exemple, pour un individu $q$ de la race pure $\mathrm{A}$ :

$$
V G E_{q}=\Sigma_{j} X_{q j A} \beta_{j A}
$$

La sélection génomique s'appuie sur le déséquilibre de liaison existant entre marqueurs et QTL. Cependant, les associations entre marqueurs et QTL, et par conséquent les effets des génotypes aux marqueurs, peuvent varier entre populations. Le modèle estimant les effets des marqueurs intra-race semble donc plus pertinent, en particulier si les populations de race pure considérées ont divergé depuis de nombreuses générations (par exemple, populations paternelles $v S$ populations maternelles) ou si la densité de marqueurs est faible (augmentant la probabilité de recombinaisons entre marqueurs et QTL). Cependant, le nombre de paramètres à estimer avec ce modèle est plus important que lorsqu'on estime un seul effet par SNP commun à toutes les races, et une population de 
Tableau 1. Synthèse des apports potentiels d'une évaluation génomique dans les schémas d'amélioration génétique porcins.

\begin{tabular}{|c|c|c|}
\hline Paramètre considéré & $\begin{array}{l}\text { Apport potentiel de la } \\
\text { sélection génomique }\end{array}$ & $\begin{array}{l}\text { Conditions nécessaires } \\
\text { / facteurs limitants }\end{array}$ \\
\hline Intervalle entre générations & Très limité à nul & \\
\hline Intensités de sélection & Limité (dégradation ?) & Coût du génotypage des candidats \\
\hline $\begin{array}{l}\text { Précision des valeurs génétiques } \\
\text { estimées }\end{array}$ & Fort & $\begin{array}{l}\text { Population de référence de taille suffisante } \\
\text { / coût }\end{array}$ \\
\hline $\begin{array}{l}\text { Sélection sur de nouveaux caractères } \\
\text { non mesurables en routine }\end{array}$ & Fort & $\begin{array}{l}\text { Disposer de phénotypes pertinents } \\
\text { / coût de constitution des populations de } \\
\text { référence }\end{array}$ \\
\hline $\begin{array}{l}\text { Prise en compte des interactions } \\
\text { génotype } \mathrm{x} \text { milieu }\end{array}$ & Fort & $\begin{array}{l}\text { Phénotypage des populations de référence en } \\
\text { milieu de production }\end{array}$ \\
\hline $\begin{array}{l}\text { Estimation de la valeur en croisement } \\
\text { d'individus de races pures (ou de } \\
\text { lignées) }\end{array}$ & Fort & $\begin{array}{l}\text { Constitution de populations de référence } \\
\text { constituées de truies parentales et de porcs } \\
\text { charcutiers } \\
\text { / modélisation statistique adéquate }\end{array}$ \\
\hline
\end{tabular}

référence de plus grande taille est donc nécessaire pour conserver une bonne précision d'estimation des effets des marqueurs (Ibanz-Escriche et al 2009). De plus, le modèle «intra-race» nécessite de connaître l'origine raciale de chaque allèle porté par les individus de la population de référence croisée, mais des erreurs d'attribution seraient en pratique inévitables, réduisant peut-être son efficacité par rapport à un modèle plus simple.

Ces deux modèles, appliqués à une population de référence croisée, permettraient donc d'estimer l'effet de substitution des allèles présents dans les races pures dans le fond génétique de la population croisée que l'on cherche à améliorer. Selon les auteurs (Ibanz-Escriche et al 2009, Kinghorn et al 2010), une partie des effets de dominance et d'épistasie s'exprimant en croisement, bien que non explicitement modélisés, seraient implicitement inclus dans ces estimations. Une meilleure modélisation des effets génétiques non additifs, sujet qui fait actuellement l'objet de travaux de recherche, permettrait sans doute d'accroître l'efficacité d'une telle approche.

Par ailleurs, l'enregistrement des performances de la population de référence dans un environnement représentatif du milieu de production permettrait d'estimer pour les individus des races pures des valeurs génomiques pour les caractères exprimés en conditions commerciales, et de s'affranchir ainsi du problème d'éventuelles interactions génotype $\mathrm{x}$ milieu. Le progrès génétique réalisé dans la population croisée à l'étage de production serait donc plus important grâce à un tel dispositif; en revanche, le progrès réalisé à l'étage de sélection serait nécessairement plus faible qu'avec des objectifs de sélection définis en race pure.
Bien que ces modèles n'aient pour l'instant pas été testés sur de vraies populations mais aient uniquement fait l'objet d'études par simulation, la possibilité de sélectionner les individus de races pures en se fondant sur des valeurs génomiques estimées à l'aide d'une population de référence croisée semble néanmoins prometteuse pour les schémas porcins. Le tableau 1 synthétise les apports potentiels d'une évaluation génomique pour les différents paramètres considérés.

\section{3 / Mise en place d'une évaluation génomique chez le porc : de l'idéal à la réalité}

Compte tenu de l'ensemble des éléments précédents, on pourrait imaginer faire évoluer les actuels dispositifs d'amélioration génétique porcins organisés autour d'un contrôle de performances des candidats et de collatéraux et d'évaluations génétiques BLUPmodèle animal vers des dispositifs fondés sur des évaluations génomiques. Dans l'idéal (figure 3), des populations de référence constituées de porcs charcutiers croisés élevés dans des conditions de production et phénotypés pour l'ensemble des caractères d'intérêt de production et de qualité permettraient d'établir les équations de prédiction de la valeur génomique des populations en sélection. Ce dispositif serait complété dans les populations maternelles par une évaluation génomique pour la prolificité et les aptitudes maternelles s'appuyant sur des populations de référence constituées de truies parentales croisées d'élevages de production.

Cependant, la mise en place effective d'un tel scénario nécessite de résoudre un certain nombre de difficultés pratiques.

\section{1 / Coût de la constitution des populations de référence et $d u$ génotypage des candidats}

On ne dispose pour l'instant pas des éléments nécessaires au dimensionnement précis des populations de référence à mettre en place en vue d'une sélection génomique chez le porc. Cependant, compte tenu des résultats de simulations, du retour d'expérience des schémas génomiques bovins laitiers, et du niveau présumé du déséquilibre de liaison entre les marqueurs de la puce porcine $60 \mathrm{~K}$ actuellement disponible, une population de référence de plusieurs milliers d'individus semble nécessaire pour atteindre des précisions satisfaisantes (c'est-à-dire au moins égales aux précisions obtenues dans les dispositifs actuels) pour une population sélectionnée dont la taille efficace est proche de 100, en particulier si l'on s'intéresse à des caractères peu héritables comme la qualité de la viande ou les aptitudes maternelles. Il est probable que les coûts de génotypage diminueront au cours des prochaines années, mais en considérant un prix approximatif de $150 €$ par individu, la constitution initiale d'une population de référence s'élèverait donc à plusieurs centaines de milliers d'euros, auxquels viendraient s'ajouter les coûts de phénotypage, évidemment dépendants de la liste des caractères considérés. De plus, une population de référence doit être renouvelée régulièrement afin de préserver la qualité de prédiction des valeurs génomiques des candidats (Solberg et al 2009). La vitesse de perte d'efficacité de la prédiction est inversement liée au niveau du déséquilibre de liaison et à la densité des marqueurs, et même si des références précises manquent encore pour l'espèce porcine, on peut néanmoins faire l'hypothèse de la validité d'une population de 
Figure 3. Organisation théorique d'un dispositif d'amélioration génétique porcin s'appuyant sur des évaluations génomiques et des populations de référence constituées d'individus croisés, dans le cas d'un croisement 3 voies de deux populations maternelles $A$ et $B$ et d'une population paternelle $C$.

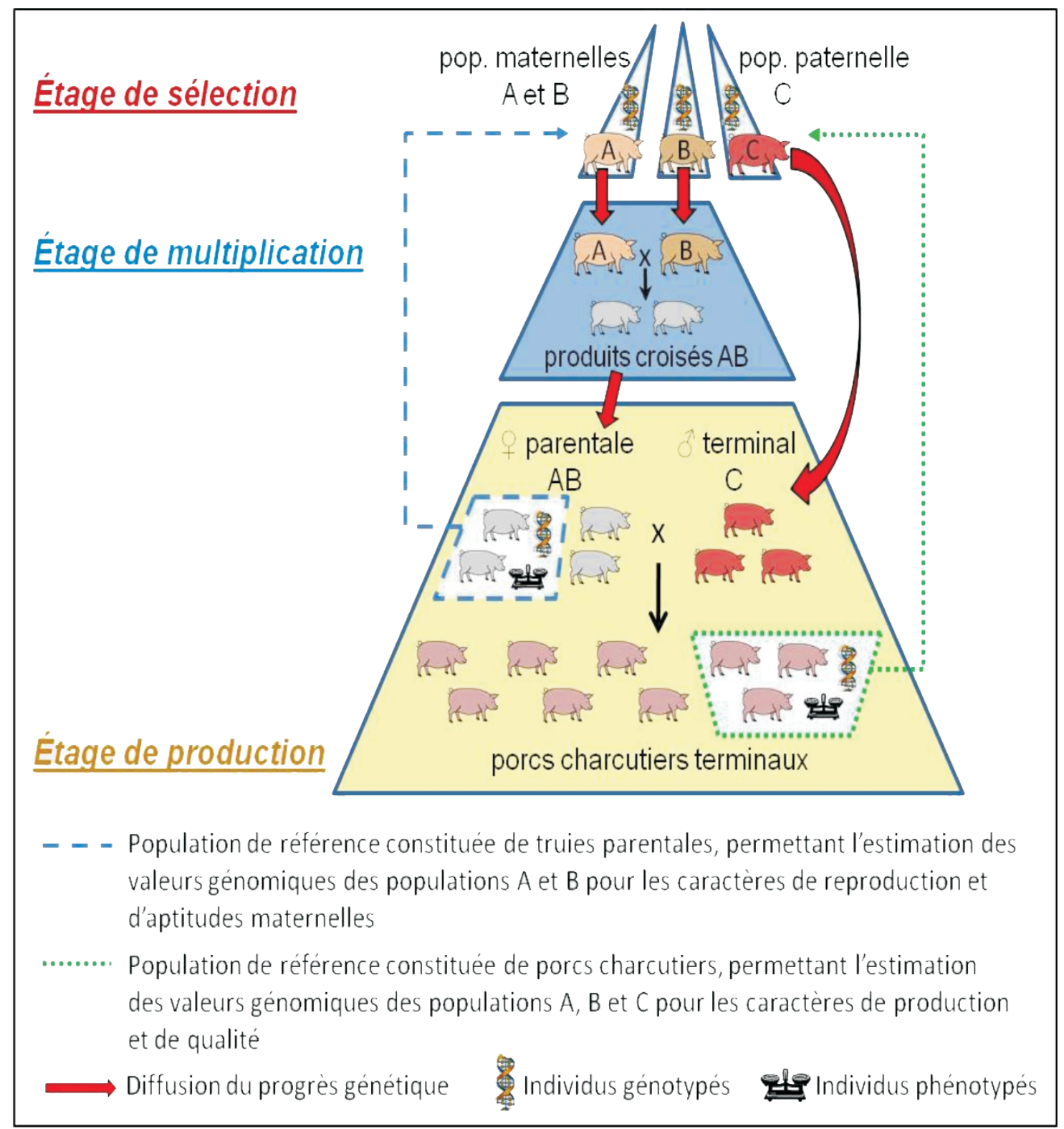

référence pendant 3 générations, ce qui impliquerait de renouveler la dépense initiale environ tous les 5 ans. De plus, ces coûts seraient approximativement doublés pour les populations maternelles, puisque les phénotypes pour les caractères de production et de reproduction ne pourraient pas être mesurés dans une même population de référence. Enfin, l'évaluation génomique d'éventuels nouveaux caractères dont le phénotypage serait incompatible avec des conditions d'élevage classiques pourrait nécessiter la constitution de populations de référence spécifiques supplémentaires.

A ces charges viendraient s'ajouter celle du génotypage des candidats à la sélection, directement liées au nombre de candidats à évaluer, et donc à l'intensité de sélection et au progrès géné- tique réalisés. Des études (Druet et Georges 2010) montrent que sous certaines conditions (par exemple, génotypage d'une partie des reproducteurs de la population pour l'ensemble des marqueurs considérés dans la population de référence), il serait possible de ne génotyper les candidats que pour quelques milliers de marqueurs informatifs répartis de manière homogène sur le génome, et «d'imputer» avec un très faible taux d'erreur leur génotype aux marqueurs manquants. Ces techniques d'imputation permettraient de diminuer sensiblement les coûts de génotypage des candidats, tout en préservant l'efficacité de l'évaluation génomique. Néanmoins, même sous l'hypothèse favorable d'un coût de $30 €$ par individu, le génotypage pour une puce de faible densité des 9200 candi- dats mâles et des 35000 candidates femelles contrôlés en ferme en 2008 dans la population collective française Large White type femelle s'élèverait par exemple à plus de $1300 \mathrm{~K} €$.

Les sommes à engager pour mettre en place une sélection génomique dans les dispositifs porcins sont donc importantes. Contrairement à la situation des schémas bovins laitiers pour lesquels les économies réalisées grâce à l'arrêt du testage sur descendance $(40000 €$ par candidat mâle testé en France) ont compensé les frais liés à l'évaluation génomique, un mode de financement reste à trouver chez le porc. Le coût des mesures actuellement réalisées sur les candidats en ferme, qui se limitent en général à des pesées et des mesures par ultrasons, est faible, et un éventuel arrêt 
du contrôle en ferme permis par une évaluation génomique ne conduirait pas à des économies substantielles. Le contrôle des collatéraux est en revanche plus coûteux, nécessitant l'équipement des élevages ou des stations en automates d'alimentation et le suivi des animaux à l'abattoir pour les mesures de qualité, avec parfois une diminution de la valeur de la carcasse en cas de prélèvement de tissus. Cependant, on peut dans une première approche considérer que le phénotypage des individus des populations de référence remplacerait celui des collatéraux, et là encore aucun report de financement vers des génotypages n'est à espérer.

Toutefois, si on tient compte du volume annuel de la production porcine $(25$ millions de porcs charcutiers ont été abattus en France en 2008), l'ensemble des coûts liés à la constitution des populations de référence, au génotypage des candidats et à l'encadrement des dispositifs serait a priori faible comparé au profit supplémentaire réalisé à l'étage de production grâce à un accroissement du progrès génétique permis par la sélection génomique. On pourrait en conséquence imaginer de financer l'augmentation du coût de la sélection par une hausse du prix de vente des reproducteurs et de la semence. Les valeurs individuelles d'une cochette et d'un verrat sont certes faibles (entre 260 et $300 €$ pour une cochette, environ $650 €$ pour un verrat, IFIP 2009), mais le nombre de reproducteurs vendus annuellement est très élevé (environ 400000 cochettes et 9400 verrats ont été vendus en France en 2008, tous types génétiques confondus, IFIP 2009), si bien que cette augmentation serait a priori modérée. Des études économiques devront cependant être conduites afin de quantifier de manière plus précise les sommes concernées et trouver le mode de financement le plus adapté à une évolution des dispositifs porcins.

\section{2 / Cas des petites populations}

De nombreuses lignées et variétés de races en sélection comptent moins de 200 femelles reproductrices, et même si la taille de la population de référence nécessaire à l'estimation de valeurs génomiques avec une précision donnée est plus faible dans les populations de petite taille efficace $(\mathrm{Ne})$, la constitution de dispositifs suffisamment puissants au sein de chacune de ces populations paraît difficile. Il semble néanmoins envisageable dans de tels cas d'utiliser des équations de prédiction des valeurs génomiques établies dans une autre race. L'efficacité d'une telle solution nécessite cependant une densité de marqueurs beaucoup plus forte que lorsque la population de référence et la population sélectionnée sont du même type génétique, afin de disposer d'associations entre marqueurs et QTL valides dans l'ensemble des populations. De Roos et al (2008) ont ainsi estimé à 300000 le nombre de SNP nécessaires à une évaluation efficace de la population bovine laitière Jersiaise à l'aide d'effets de marqueurs estimés en Holstein. Il est possible que la puce porcine de 60000 SNP actuellement sur le marché soit insuffisante pour de telles applications, mais des puces plus denses seront certainement bientôt disponibles. Par ailleurs, une représentation, même faible, d'individus de la population à évaluer au sein de la population de référence semble permettre d'améliorer sensiblement la précision d'évaluation (Harris et al 2008, de Roos et al 2008), et les organisations de sélection détentrices de petites populations seront donc vraisemblablement amenées à collaborer afin de constituer des dispositifs multiraciaux.

\section{3 / Partages des ressources}

Cette mise en commun de ressources ne se limitera vraisemblablement pas aux seules petites populations. En effet, compte tenu du nombre élevé de populations porcines sélectionnées (par exemple, en France, 5 grandes races, une dizaine de variétés de ces races et une quinzaine de lignées composites), de la variété des truies parentales et des types de porcs charcutiers produits, des capacités de phénotypage limitées pour certains caractères (efficacité alimentaire) et des sommes à engager, il semble peu probable de pouvoir constituer et entretenir des populations de référence spécifiques pour un grand nombre de populations sélectionnées ou de croisements pratiqués. Une mise en commun des phénotypes et génotypes de populations génétiquement proches détenues par plusieurs Organisations de Sélection semble donc nécessaire, permettant d'augmenter la puissance des dispositifs tout en limitant les coûts engagés par chaque partenaire. Des collaborations internationales sont également envisageables, à l'image du projet Eurogenomics destiné à mettre en commun les phénotypes et génotypes de 16000 taureaux Holstein de 6 pays européens (Lund et al 2010). En pratique, un tel partage des ressources suppose cependant une homogénéité des phénotypes mis en commun par les partenaires potentiels, ce qui était le cas chez les bovins laitiers de par l'existence de l'évaluation génétique internationale INTERBULL. Celle-ci n'a malheureusement pas son équivalent actuellement chez le Porc, espèce dans laquelle un effort préalable serait donc nécessaire.

\section{Conclusions}

La sélection génomique offre plusieurs pistes d'amélioration de l'efficacité des schémas d'amélioration génétique porcins, bien qu'il soit peu probable que sa mise en place entraîne des mutations aussi profondes que les changements auxquels nous assistons dans les schémas bovins laitiers, principalement avec l'arrêt du testage sur descendance. Les perspectives les plus intéressantes semblent être la possibilité d'estimer plus précisément la valeur génétique des individus pour les caractères peu héritables et non observables sur les candidats (qualité de la viande, efficacité alimentaire, aptitudes maternelles) et de sélectionner plus efficacement les individus de race pure pour les caractères exprimés en croisement et dans des conditions de production.

Bien que cette méthodologie permette d'estimer la valeur génétique de candidats sans performance propre et sans performance d'apparenté, l'enregistrement de phénotypes pour les caractères que l'on souhaite améliorer reste tout de même primordial afin d'établir les équations de prédiction des valeurs génomiques. Les stations publiques porcines, bien que les conditions d'environnement $y$ soient sensiblement différentes de celles des élevages commerciaux, seraient naturellement un outil de choix pour la constitution des populations de référence. Elles permettent en effet de mesurer de manière précise les performances des individus pour un grand nombre de caractères, et la nécessité de leur maintien dans des schémas d'amélioration génétique s'appuyant sur la sélection génomique est donc primordiale. La création d'un réseau d'élevages de référence dont la fonction serait analogue à celles des stations pour les caractères de reproduction et d'aptitudes maternelles semble également nécessaire.

De nombreuses incertitudes demeurent cependant quant aux modalités de mise en œuvre de la sélection génomique dans les schémas porcins, qui devraient être levées par les prochaines avancées technologiques (puces plus denses et à moindre coût) et méthodologiques (meilleure prise en compte des effets génétique non additifs, mélanges de populations). Des études par simulations devront également être conduites afin de déterminer la manière optimale de réorganiser les schémas d'amélioration génétique porcins autour de cette nouvelle méthodologie. 


\section{Références}

Dekkers J.C.M., 2007. Marker assisted selection for commercial crossbred performance. J. Anim. Sci., 85, 2104-2114.

De Roos A.P.W., Hayes B.J., Spelman R., Goddard M.E., 2008. Linkage disequilibrium and persistence of phase in Holstein Friesian, Jersey and Angus cattle. Genetics, 179, 1503 1512 .

Druet T., Georges M., 2010. A hidden Markov model combining linkage and linkage disequilibrium information for haplotype reconstruction and Quantitative Trait Locus fine mapping. Genetics, 184, 789-798.

Du F.X., Clutter A.C., Lohuis M.M., 2007. Characterizing linkage disequilibrium in pig populations. Int. J. Biol. Sci., 3, 166-178.

Fritz S., Colleau J.J., Druet T., Boscher M.Y., Rossignol M.N., Malafosse A., Boichard D., 2003. Mise en place d'une Sélection Assistée par Marqueurs dans les trois principales races bovines laitières françaises. Renc. Rech. Rum., 10, 53-56.

Guillaume F., Boichard D., Ducrocq V., Fritz S., 2011. Utilisation de la sélection génomique chez les bovins latiier. .In : Numéro spécial, Amélioration génétique. Mulsant P., Bodin L., Coudurier B., Deretz S., Le Roy P., Quillet E., Perez J.M. (Eds). INRA Prod. Anim., 24, 363368.

Habier D., Götz K.U., Dempfle L., 2007. Estimation of genetic parameters on test stations using purebred and crossbred progeny of sires of the Bavarian Piétrain. Livest. Sci., 107, 142-151.

Harris B.L., Johnson D.L. Spelman R.J., 2008. Genomic selection in New Zealand and the implications for national genetic evaluation. Proc. Interbull Meeting, Niagara Falls, Canada.http://www.cabi.org/cabdirect/FullText PDF/ 2010/20103193083.pdf
Hu Z.L., Reecy J.M., 2007. Animal QTLdb: beyond a repository - A public platform for QTL comparisons and integration with diverse types of structural genomic information. Mamm. Genome, 18, 1-4.

Ibanz-Escriche N., Fernando R.L., Toosi A., Dekkers J.C.M., 2009. Genomic selection of purebreds for crossbred performance. Genet. Sel. Evol., 41, 12-21.

IFIP, Institut du Porc, 2009. Le porc par les chiffres. Ed. IFIP, France, $54 \mathrm{p}$

Kinghorn B.P., Hickey J.M., van der Werf J.H.J., 2010. Reciprocal recurrent genomic selection for total genetic merit in crossbred individuals. Proc $9^{\text {th }}$ WCGALP, August 1-6, Leipzig, Germany. Comm. 36

Lund M.S., de Roos A.P.W., de Vries A.G., Druet T., Ducrocq V., Fritz S., Guillaume F., Guldbrandtsen B., Liu Z., Reents R., Schrooten C., Seefried M., Su G., 2010. Improving genomic prediction by EuroGenomics collaboration. Proc $9^{\text {th }}$ WCGALP, August 1-6, Leipzig, Germany. Comm. 0880.

Lutaaya E., Misztal I., Mabry J.W., Short T. Timm H.H., Holzbauer R., 2001. Genetic parameter estimates from joint evaluation of purebreds and crossbreds in swine using the crossbred model. J. Anim. Sci., 79, 3002-3007.

Luther H., Vögeli P., Hofer A., 2009. Increasing genetic E. coli F18 resistance in Swiss pigs. Proc. 60 ${ }^{\text {th }}$ EAAP Meeting, 24-27 August, Barcelona, Spain. Comm. 18-03.

Maignel L., Tribout T., Boichard D., Bidanel J.P., Guéblez R., 1998. Analyse de la variabilité génétique des races porcines Large White, Landrace Français et Piétrain, sur la base de l'information généalogique. Journ. Rech. Porcine Fr., 30, 109-116.

Merks J.W.M. 1989. Genotype $\times$ environment interactions in pig breeding programmes.
VI. Genetic relations between performances in central test, on-farm test and commercial fattening. Livest. Prod. Sci., 22, 325-339.

Meuwissen T.H.E., 2009. Accuracy of breeding values of unrelated individuals predicted by dense SNP genotyping. Genet. Sel. Evol., 41, 35.

Sidani C., Astruc J.M., Baelden M., Barillet F., Bibé B., Bonnot A., Boscher M.Y. Bouchel D., Bouffartigue B., Bouix J., Brochard M., Dion F., Francois D., Jouhet E., Jullien E., Leymarie C.R., Moreno C., Orlianges M., Palhière I., Perret G., Raoul J., Raynal A., Tiphine L., Tribon P., 2010. The french ovine scrapie plan: results and prospects. Proc. $9^{\text {th }}$ WCGALP, August 1-6, Leipzig, Germany. Comm. 412.

Schwob S., Riquet J., Bellec T., Kernaleguen L., Tribout T., Bidanel J.P., 2009. Mise en place d'un programme de sélection assistée par marqueurs dans la population sino-européenne Duochan. Journ. Rech. Porcine Fr., 41, 29-30.

Solberg T.R., Sonesson A.K., Woolliams J.A., Odegard J., Meuwissen T.H.E., 2009. Persistence of accuracy of genome-wide breeding values over generations when including a polygenic effect. Genet. Sel. Evol., 41, 53.

Uimari P., Tapio M., 2011. Extent of linkage disequilibrium and effective population size in Finnish Landrace and Finnish Yorkshire pig breeds. J. Anim. Sci., 89, 609-614.

Van der Steen H.A.M., Prall G.F.W., Plastow G.S., 2005. Application of genomics to the pork industry. J. Anim. Sci., 83 (E. Suppl.), E1-E8.

Wei M., Van der Steen H.A.M., 1991 Comparison of reciprocal recurrent selection with pure-line selection systems in animal breeding (a review). Anim. Breed. Abstr., 59, 281-298.

\section{Résumé}

L'évaluation génomique est une nouvelle méthode d'estimation de la valeur génétique des animaux d'élevage qui est en train de révolutionner l'organisation des schémas d'amélioration génétique bovins laitiers. La récente disponibilité d'une puce porcine de 60000 marqueurs SNP permet d'envisager la mise en place de cette méthodologie chez le porc. Dans cette espèce, les perspectives les plus intéressantes de l'évaluation génomique semblent être la possibilité d'améliorer les populations pour des caractères non mesurables en routine, d'augmenter la précision des valeurs génétiques estimées, et de sélectionner les individus de l'étage de sélection pour leur valeur en croisement et en milieu de production. Les coûts de mise en oeuvre d'une sélection génomique chez le porc seraient élevés, en raison principalement de la taille des populations de référence nécessaires à une évaluation précise, du nombre élevé de candidats à génotyper, et de la variété des populations et lignées sélectionnées. Le partage de ressources entre Organisations de Sélection et le recours aux techniques d'imputation génotypiques permettraient de maîtriser ces coûts.

\section{Abstract}

\section{Potential applications of genomic selection in pig breeding schemes}

Genomic evaluation, a new methodology to estimate the breeding value of livestock, is currently revolutionizing dairy cattle breeding, and its implementation in pigs is being considered. In this species, genomic selection could be used to improve populations for traits too costly to be recorded routinely, to increase the accuracy of the estimated breeding values, and to select the nucleus populations for their crossbred value expressed in a commercial environment. The implementation of genomic selection in pigs would be costly, mainly because of the size of the reference populations required to estimate accurate breeding values, the high number of candidates that should be genotyped, and the large number of selected lines. The sharing of reference populations by several breeding organizations and the use of genotype imputation techniques would help reduce the implementation costs.

TRIBOUT T., 2011. Perspectives d'application de la sélection génomique dans les schémas d'amélioration génétique porcins. In : Numéro spécial, Amélioration génétique. Mulsant P., Bodin L., Coudurier B., Deretz S., Le Roy P., Quillet E., Perez J.M. (Eds). INRA Prod. Anim., 24, 369-376. 